\title{
A Novel Approach Based on Spatio-temporal Features and Random Forest for Scar Detection Using Cine Cardiac Magnetic Resonance Images
}

\author{
Sara Moccia ${ }^{1,2}$, Alessandro Cagnoli ${ }^{3}$, Chiara Martini ${ }^{4}$, Giuseppe Moscogiuri ${ }^{5}$, Mauro Pepi ${ }^{5}$, \\ Emanuele Frontoni ${ }^{1}$, Gianluca Pontone ${ }^{5}$, Enrico Gianluca Caiani ${ }^{3}$ \\ ${ }^{1}$ Department of Information Engineering, Università Politecnica delle Marche, Ancona, Italy \\ ${ }^{2}$ Department of Advanced Robotics, Istituto Italiano di Tecnologia, Genoa, Italy \\ ${ }^{3}$ Department of Electronics, Information and Bioengineering, Politecnico di Milano, Milan, Italy \\ ${ }^{4}$ Diagnostic Department, Azienda Ospedaliera-Universitaria di Parma, Parma, Italy \\ ${ }^{5}$ Department of Cardiovascular Imaging, Centro Cardiologico Monzino IRCCS, Milan, Italy
}

\begin{abstract}
Aim. To identify the presence of scar tissue in the left ventricle from Gadolinium $(G d)$-free magnetic resonance cine sequences using a learning-based approach relying on spatiotemporal features. Methods. The spatial and temporal features were extracted using local binary patterns from (i) cine end-diastolic frame and (ii) two parametric images of amplitude and phase wall motion, respectively, and classified with Random Forest. Results. When tested on 328 cine sequences from 40 patients, a recall of $70 \%$ was achieved, improving significantly the classification resulting from spatial and temporal features processed separately. Conclusions. The proposed approach showed promising results, paving the way for scar identification from $G d$-free images.
\end{abstract}

\section{Introduction}

Ischemic heart disease represents the main cause $(>60 \%$ of cases) of dilatated cardiomyopathy (DCM), which affects 5 in 100,000 adults and is the third leading cause of heart failure in the United States. [1] The presence of ischemic scar tissue in the left ventricle (LV) is usually assessed with Magnetic Resonance with late Gadolinium (Gd) enhancement (CMR-LGE). In CMR-LGE, Gd is injected intravenously and deposits in the nonviable tissue, resulting in hyper-enhanced areas in CMR-LGE images [2]. CMR-LGE is, however, highly contraindicated in patients with severe kidney disorders and, more dramatically, a recent study has shown that Gd may represent a long-term risk factor even in subjects with normal renal function [3].

As nonviable scar tissue modifies the LV contractile properties, its identification from images obtained by steady-state free precession (SSFP) cine CMR pulse sequences could be explored [4]. On this matter, in [5] a machinelearning (ML) classifier was used to identify the presence of scar tissue in LV sectors using Local Binary Patterns (LBP), computed from the cine dynamic images using a $2 \mathrm{D}+\mathrm{t}$ approach, as textural descriptor. With the same goal, in [6] temporal features were extracted using optical flow and classified using auto-encoders. Both [5] and [6] rely on spatio-temporal learning paradigms to tackle the low contrast of scar tissue in cine frames. In both cases, however, the feature size easily grows because features are extracted from all frames in the cardiac cycle, thus posing issues relevant to the classifier overfitting.

Inspired by the previous considerations, we hypothesized that the (spatial) texture information encoded in the end diastolic (ED) frame could be enriched by a compact set of features extracted along the temporal dimension, to fully exploit the wall-motion temporal continuity encoded in cine dynamic images. 


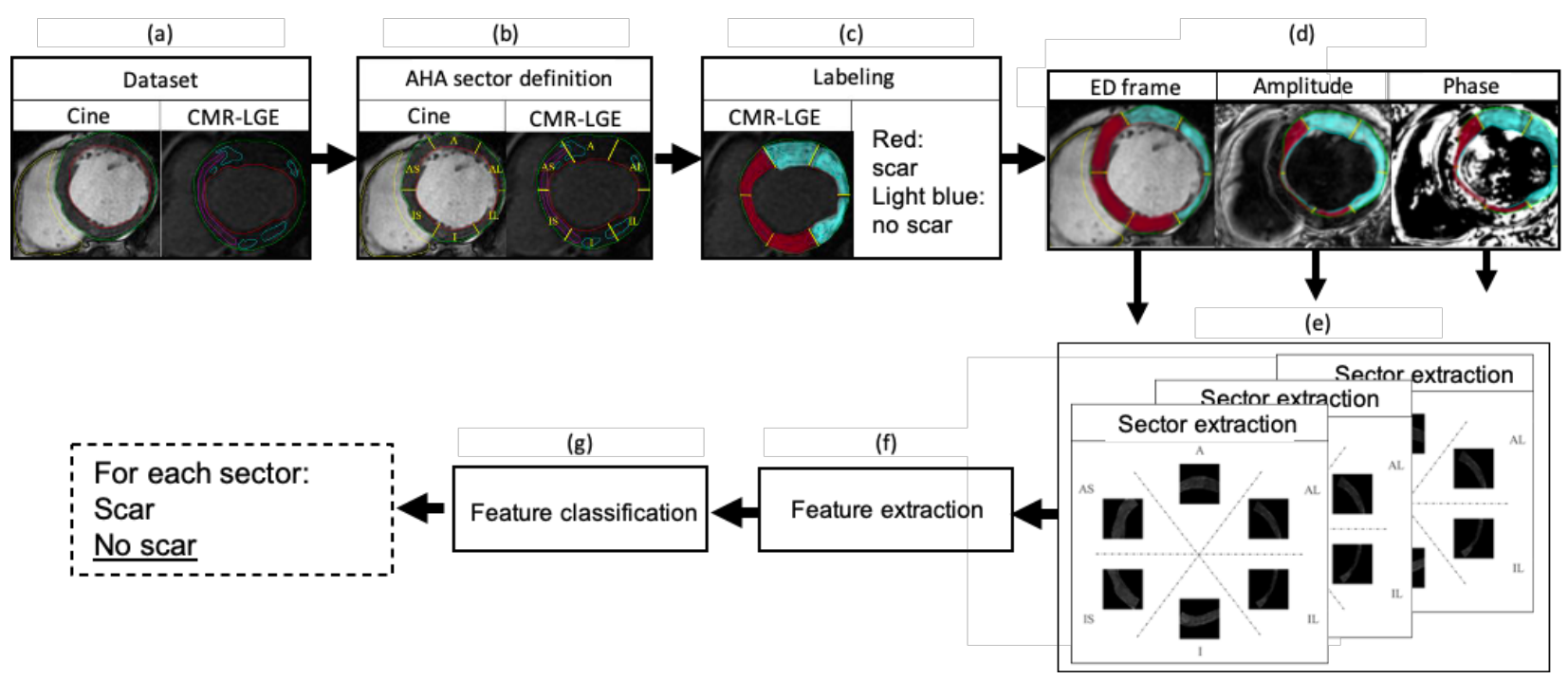

Fig. 1: Workflow of the proposed approach for scar identification from (a) cine images, with the CMR-LGE used only as gold-standard reference. First, (b) the left ventricle is divided into six AHA sectors, applied to both the cine and CMR-LGE. (d) In addition to the end diastolic (ED) cine frame, two parametric images (phase and amplitude) are computed from the cine dynamic images. (e-f) Textural features are extracted, for each sector, from the ED, amplitude and phase images, and (g) classified using Random Forest, to obtain a classification of scar/ no scar for each sector, compared to the gold standard.

Accordingly, our aim was to propose an innovative ML approach for the identification of scar tissue in LV from dynamic cine images and test its feasibility and accuracy in classification of LV sectors compared to an expert gold standard (GS).

\section{Methods}

\subsection{Experimental design}

The cine dynamic images (20 images per cardiac cycle, SSFP sequence) acquired in 40 patients diagnosed with ischemic DCM at Centro Cardiologico Monzino (Milan, Italy) were retrospectively selected. All datasets were acquired using a 1.5 Tesla scanner (Discovery MR 450, GE Healthcare, Waukesha, WI) with a 32-channel phased array receiver coil. The corresponding CMR-LGE images were available for GS assessment, performed by an expert cardiologist, which provided the myocardial contours and scar tracings on the ED CMR-LGE. Based on these tracings, and considering the division of the myocardium into 6 sectors according to the AHA model [7], for each sector the scar traced inside the myocardium was considered. To avoid counting scars overlapping multiple sectors, but with few pixels, if the ratio between the scar and total myocardium area in a sector was lower than $10 \%$, that sector was excluded. For each of the included sectors, the GS resulted into a binary classification of scar/no scar, used for both training and testing the ML approach.

\subsection{Image processing}

Figure 1 shows a schematization of the proposed workflow. First, in each cine frame, the LV was divided in 6 sectors, according to the AHA model [7].
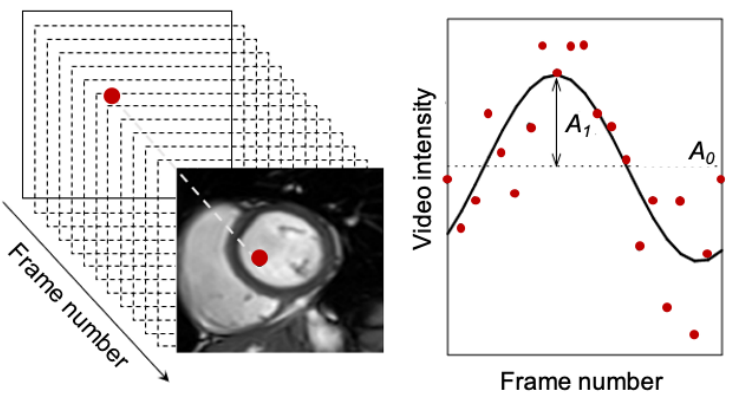

Fig. 2: Parametric image generation process. The $A 1$ and AO parameters are highlighted. 
For each cine loop, parametric images relevant to amplitude and phase of pixel video-intensity oscillation over the cardiac cycle were computed [8]. Specifically, for each pixel position, its values were extracted throughout the cardiac cycle to create time series of video intensity.

Each time series was then fitted into a sinusoidal function by using standard least squares analysis. The amplitude $\left(A_{1}\right)$ of the first harmonic of the best-fit curve was calculated, as shown in Fig. 2, and its normalized value ( $A_{1} / A_{0}$, being $A_{o}$ the curve mean) displayed in each pixel, resulting in the amplitude parametric image. The phase $(\varphi)$ parametric image was obtained in the same way, by displaying the phase value of the best fit curve.

The idea behind using these images was to capture the myocardial wall motion information, and possible akinesis/ diskinesis, in a simple still frame: the $A_{1} / A_{0}$ results in higher intensity in pixels changing between scar and healthy tissue during the endocardial contraction/ relaxation, while the $\varphi$ carries information about myocardial synchronicity.

The feature descriptors were extracted at:

- Spatial level: from the sectors in the ED frame of the cine loop;

- Temporal level: from the sectors extracted from both the $A_{1} / A_{0}$ and $\varphi$ parametric images.

Following [7], the histogram $\left(H_{L B P}\right)$ of the uniform rotation invariant LBP was selected as feature descriptor. The LBP radius and number of neighbors were set to $(1,8),(2,16),(3,24)$, following the standard LBP implementation.

For LBP feature classification, Random Forest (RF) was chosen [9] as it allows good classification performance at a low training computational cost. During the training phase, the RF hyperparameters (i.e., number of trees and tree depth) were set via grid search and 5-fold cross validation on the training set.

The grid search for the number of trees and the tree depth was set to $(5,10,15,20,30,40)$ and $(2,3,4,5)$, respectively. Both the number of trees and tree depth were kept low to prevent overfitting.

Classification performance was assessed using 3-fold cross-validation for robust testing. All the analyses were performed on an Intel ${ }^{\circledR}$ Core $^{\mathrm{TM}}$ i78700CPU (3.2 GHz, $6 \mathrm{MB}$ cache, 8 cores) computer. The feature extraction and classification were implemented with scikitlearn (https://scikit-learn.org/stable/index.html).

\subsection{Statistical analysis}

The area $(A U C)$ under the receiver operating characteristic (ROC) curve, the classification recall $(R e c)$, precision $(P r e c)$ and $\mathrm{F} 1$-score $(F 1)$ were computed.

In order to understand the potential, separately and altogether, of the extracted features, classification and comparison with GS was performed considering:

1) Spatial feature (2D), as $H_{L B P}$ from the ED cine frame;

2) Temporal feature (A1/A0), as $H_{L B P}$ from the amplitude parametric image;

3) Spatio-temporal features (2D+A1/A0), as concatenation of the $H_{L B P}$ from the ED cine frame and from the amplitude parametric image;

4) Spatio-temporal features as concatenation of the $H_{L B P}$ from the ED cine frame and from both the amplitude and phase parametric images $(2 \mathrm{D}+\mathrm{A} 1 / \mathrm{A} 0+\varphi)$.

The non-parametric Kruskal-Wallis test was used for multiple comparisons between the performances of the different sets of features. The Mann-Whitney test with Bonferroni correction was used as post-hoc method.

Table 1: Median (inter-quartile range) classification results, expressed in $\%$, for the tested feature sets. $*=\mathrm{p}<0.05$ vs $2 \mathrm{D}+\mathrm{A} 1 / \mathrm{A} 0+\varphi$

\begin{tabular}{l|ccc} 
& Rec & Prec & $F 1$ \\
\hline 2D* & $65(7)$ & $66(7)$ & $65(7)$ \\
A1/A0* & $62(4)$ & $62(4)$ & $68(4)$ \\
2D + A1/A0 & $68(2)$ & $68(2)$ & $68(2)$ \\
$2 \mathrm{D}+\mathrm{A} 1 / \mathrm{A} 0+\varphi$ & $\mathbf{7 0 ( 2 )}$ & $\mathbf{6 9 ( 1 )}$ & $\mathbf{6 9 ( 2 )}$
\end{tabular}




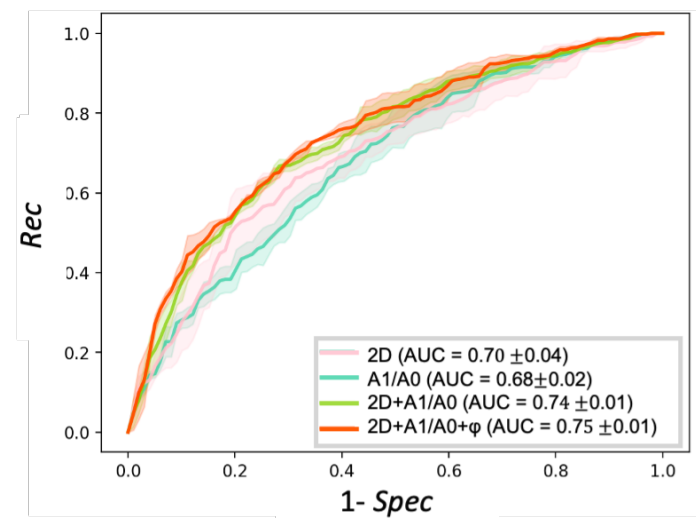

Fig. 3: Receiver operating characteristic curve for the tested feature sets. The area (AUC) under the curve is reported in terms on mean \pm standard deviation.

\section{Results}

A total of 328 cine loops, each relevant to a CMR slice, together with the corresponding LGE images, were available from the 40 patients included in this study.

A total of 1968 AHA sectors were available $(328 * 6)$ for GS assessment. Based on the $10 \%$ area ratio condition, 1458 sectors were considered, of which 511 had scar, and 947 had not. Accordingly, prior to 3-fold crossvalidation, the number of sectors was balanced, thus resulting in a final dataset of 1022 sectors for features extraction and classification.

Figure 3 shows the ROC curves resulting from the tested feature sets. As shown in Table 1, the best results were achieved when classifying the spatio-temporal features including $\varphi$, with improved Rec compared to the other feature sets.

\section{Discussion}

The $2 \mathrm{D}+\mathrm{A} 1 / \mathrm{A} 0+\varphi$ feature set, as a combination of both spatial and temporal features, showed the best results in terms of classification of sectors with scar. Exploiting parametric images to encode the wall-motion temporal information significantly enhanced the classification performance, while keeping the feature dimension low with respect to extracting features from all the frames in a cine sequence. The worst results were obtained both when spatial (2D) and temporal (A1/A0) features were processed separately.

Future improvements will focus on more advanced classification solutions based on deep learning, which already proved to be valuable for CMR-LGE [10].

\section{Conclusion}

A ML approach based on spatio-temporal features extracted from cine CMR images was proposed to obtain information on scar presence. Promising results were obtained using amplitude and phase parametric images to represent wall motion. This work represents a promising step toward a helpful processing system to support scar tissue identification from Gd-free images.

\section{References}

[1] McKenna W, et al. Classification, epidemiology, and global burden of cardiomyopathies. Circ Res 2017;121: 722-30.

[2] Mewton N, et al. Assessment of myocardial fibrosis with cardiovascular magnetic resonance. J Am Coll Cardiol 2011; 57: 891-903.

[3] McDonald RJ, et al. Gadolinium deposition in human brain tissues after contrast-enhanced MR imaging in adult patients without intracranial abnormalities. Radiology 2017;285: 546-54.

[4] Rajiah P, et al. MR imaging of myocardial infarction. RadioGraphics 2013; 33: 1383-1412.

[5] Larroza, A., et al. Texture analysis of cardiac cine magnetic resonance imaging to detect nonviable segments in patients with chronic myocardial infarction. Medical Physics 2018; 45: 1471-80.

[6] Zhang N, et al. Deep learning for diagnosis of chronic myocardial infarction on nonenhanced cardiac cine MRI. Radiology 2019; 291: 606-17.

[7] Mewton N, et al. Comparison of visual scoring and quantitative planimetry methods for estimation of global infarct size on delayed enhanced cardiac MRI and validation with myocardial enzymes", Eur J Radiol 2011; 78(1): 87-92

[8] Caiani EG et al. The role of still-frame parametric imaging in magnetic resonance assessment of left ventricular wall motion by non-cardiologists. J Cardiovasc Magn Reson 2004; 6:619-25.

[9] Oshiro, T et al. How many trees in a Random Forest? MLDM. Springer, 2012; 154-168.

[10] Moccia S., et al. Development and testing of a deep learningbased strategy for scar segmentation on CMR-LGE images, MAGMA 2019; 32: 187-195.

Address for correspondence:

Prof. Enrico G. Caiani

DEIB, Politecnico di Milano

enrico.caiani@polimi.it 